\title{
Note on the Relative Efficiencies of Gas and Liquid Chromatographic Columns
}

\author{
M. J. E. Golay \\ The Perkin-EImer Corporation, Norwalk, Conn. 06856, USA
}

Some 17 years ago, when trying to assess the intrinsic performance of the gas chromatographic columns of those days, it became tempting to pick as a mathematically tractable model of a column an empty tube with its inner walls coated with a liquid retentive material suitably dispersed on a large porous area and of such thinness that the diffusion time within that liquid of the molecules so retainred could be neglected. Typically a $0.01 \mathrm{~cm}$ radius tube with a capacity (partition) ratio (k) of 3 could be postulated. A primitive theory indicated then that such a tube would have an HETP of the order of $0.008 \mathrm{~cm}$ so that a carrier gas velocity of $40 \mathrm{~cm} / \mathrm{sec}$ could cause a component so retained to traverse a $4 \mathrm{~cm}$ length of such a tube, representing 500 plates, in $0.4 \mathrm{sec}$, while the pressure drop required for this passage, assuming $2 \cdot 10^{-4}$ poise for the carrier gas viscosity, was

$\Delta \mathrm{p}=\frac{8 \cdot 2 \cdot 10^{-4} \text { poise } \cdot 4 \mathrm{~cm} \cdot 4 \mathrm{~cm} / \mathrm{sec}}{(0.01 \mathrm{~cm})^{2}}=0.25 \mathrm{~atm}$.

It is well known that the then existing columns capable of yielding a 500-plate separation required the order of 10-times the pressure drop and 1000-times the elution time, so that they appeared to be four orders of magnitude less efficient than the model chosen. This led naturally to the idea of making gas chromatographic columns out of open and coated tubes, and the open tubular columns of today are within a fraction of an order of magnitude of the theoretically optimal efficiency.

When one tries to assess the efficiency of today's liquid chromatographic columns one obtains an even more dramatic disparity between their performance and the calculated theoretical performance. Thus, postulate an open retentive tube with a diameter of $1 \mu \mathrm{m}$; its HETP is of the order of $0.8 \mu \mathrm{m}$ so that a $0.04 \mathrm{~cm}$ of such a tube should yield 500 theoretical plates. With a liquid diffusion of the order of $3 \cdot 10^{-6} \mathrm{~cm}^{2} / \mathrm{sec}$ the optimal velocity will be $0.04 \mathrm{~cm} / \mathrm{sec}$, so that the time of analysis for components retained with a capacity (partition) ratio of 3 should be 4 seconds. With a viscosity of $10^{-2}$ poise, the pressure drop in this minuscule tube will be

$\Delta \mathrm{p}=\frac{8 \cdot 10^{-2} \text { poise } \cdot 0.04 \mathrm{~cm} \cdot 0.04 \mathrm{~cm} / \mathrm{sec}}{\left(10^{-4} \mathrm{~cm}\right)^{2}}=1.3 \cdot 10^{-2} \mathrm{~atm}$

These are analysis time and pressure respectively 2.5 and 4.5 orders of magnitude smaller than those required for today's liquid chromatographic columns with 500 plates, so that we are confronted by a seven order of magnitude discrepancy between the the theoretical and the actual performance of liquid chromatographic columns. This means a challenge very much greater than the one facing us 17 years ago, but how shall we meet this challenge?

To do what we did for gas chromatographic columns and resort to open tubes of sub-micron size seems impractical on several counts. Such tubes will be difficult to make and the behavior of polar liquids in them may have surprises in store for us, possibly the formation of polymerized water or other liquids such as already reported by some researchers but not generally accepted. Also, the very small throughput of such columns will make demands on detector sensitivity which may not be met soon. Perhaps the likely compromise will be very small columns with roughened walls packed with hard center spiked spheres of micron size coated with a thin layer of retentive material - the smallness of the columns to minimize the effect of multipaths on the HETP; the roughened walls to minimize the "wall effect" of smooth walls which permits a faster liquid flow along them than in the column center by providing larger passages; the spiked spheres to make the size of the passages more com. mensurate with the sphere size, and the thin coats of retentive material to minimize the diffusion time of retained molecules in the fixed phase.

Received: April 25, 1973 Accepted: May 3,1973 